\title{
Editorial
}

\section{Terahertz as a Frontier Area for Science and Technology}

\author{
Akinori Irizawa ${ }^{1}$, Stefano Lupi ${ }^{2,3}$ and Augusto Marcelli ${ }^{3,4, *(1)}$ \\ 1 The Institute of Scientific and Industrial Research (ISIR), Osaka University, 8-1 Mihogaoaka, Ibaraki, \\ Osaka 5670047, Japan; irizawa@sanken.osaka-u.ac.jp \\ 2 Department of Physics, Sapienza University, P.le Aldo Moro 5, 00185 Rome, Italy; stefano.lupi@roma1.infn.it \\ 3 Istituto Nazionale di Fisica Nucleare-Laboratori Nazionali di Frascati (INFN-LNF), Via Enrico Fermi 54, \\ 00044 Frascati, Italy \\ 4 International Centre for Material Science Superstripes, RICMASS, Via dei Sabelli 119A, 00185 Rome, Italy \\ * Correspondence: augusto.marcelli@lnf.infn.it
}

check for

updates

Citation: Irizawa, A.; Lupi, S.;

Marcelli, A. Terahertz as a Frontier

Area for Science and Technology.

Condens. Matter 2021, 6, 23. https://

doi.org/10.3390/condmat6030023

Received: 7 June 2021

Accepted: 22 June 2021

Published: 28 June 2021

Publisher's Note: MDPI stays neutral with regard to jurisdictional claims in published maps and institutional affiliations.

\begin{abstract}
Recent theoretical and experimental research is triggering interest to technologies based on radiation in the region from $\sim 0.1$ to 20 Terahertz (THz). Today, this region of the electromagnetic (e.m.) spectrum is a frontier area for research in many disciplines. The technological roadmap of the $\mathrm{THz}$ radiation considers outdoor and indoor communications, security, drug detection, biometrics, food quality control, agriculture, medicine, semiconductors, and air pollution, and demands high-power and sub-ps compact sources, modern detectors, and new integrated systems. There are still many open questions regarding working at $\mathrm{THz}$ frequencies and with $\mathrm{THz}$ radiation. In particular, we need to invest in new methodologies and in materials exhibiting the unusual or exotic properties of THz. This book contains original papers dealing with some emerging THz applications, new devices, sources and detectors, and materials with advanced properties for applications in biomedicine, cultural heritage, technology, and space.
\end{abstract}

Keywords: THz; FEL; coherent synchrotron radiation; waveguides; $\mathrm{THz}$ detector; THz imaging

\section{Introduction}

Photonics is the physical science describing the generation, the detection, the manipulation, and the application of many different devices of light. Photonics, combined with micro- and nano-electronics, biotechnology, and/or nanotechnology, is emerging as a strategic research and technological area as addressed by the United Nations which celebrated 2015 as the International Year of Light. Hence, the continuous development of photonic technologies needs a deeper understanding of light-matter interactions, the implementation of new concepts, and the synthesis of novel materials with tailored properties [1]. Following the development of laser-based Terahertz (THz) time-domain spectroscopy in the 1980s and 1990s, the field of THz science and technology expanded rapidly and now involves 'real world' applications [2-4]. The new approaches led to multidisciplinary research, combining photonics modeling with long-term strategic aims towards challenging applications such as spintronics, bio-photonics, and many unique devices that can be manufactured with the currently available technologies; others will soon appear, thanks to emerging technologies.

The spectacular advancements observed in the last decade has led to an increasing interest in THz technology in the effort to harness the power of thermal radiation in the region from $\sim 100 \mathrm{GHz}$ to $20 \mathrm{THz}\left(3.3-670 \mathrm{~cm}^{-1}\right.$ wavenumber, $3 \mathrm{~mm}-15 \mu \mathrm{m}$ wavelength, $0.4-83 \mathrm{meV}$ photon energy). The interest shares some uniquely attractive characteristics of the $\mathrm{THz}$ radiation with unique application possibilities [2,5].

The $\mathrm{THz}$ region of the electromagnetic spectrum is a frontier area for research in physics, chemistry, biology, materials science, and biomedicine. Indeed, small molecules rotate at $\mathrm{THz}$ frequencies; biologically important collective modes of proteins DNA and RNA vibrate at $\mathrm{THz}$ frequencies; frustrated rotations and collective modes cause polar 
liquids such as water to absorb at $\mathrm{THz}$ frequencies; electrons in semiconductors and their nanostructures resonate at $\mathrm{THz}$ frequencies; electrons in highly excited atomic Rydberg states orbit at $\mathrm{THz}$ frequencies; superconducting energy gaps fall at $\mathrm{THz}$ frequencies; and gaseous and solid-state plasmas also oscillate at $\mathrm{THz}$ frequencies, therefore this radiation can be used to study and control an extraordinary vast number of materials, fundamental systems, and phenomena [2,5-9].

The roadmap for the development of $\mathrm{THz}$ technologies $[2,10,11]$ considers applications in the fields of outdoor and indoor communications, security, drug detection, biometrics, food quality control, agriculture, medicine, semiconductors, and air pollution. Their exploitation and realization demand high-power compact $\mathrm{THz}$ sources, more sensitive detectors, and more functional integrated $\mathrm{THz}$ systems.

\section{Discussion}

Sources of high-quality radiation in the $\mathrm{THz}$ region of the e.m. spectrum have been scarce [12], but this "THz gap", after continuous research efforts, has been filled by a wide range of new technologies ranging from accelerated relativistic electrons [13,14], to high-power femtosecond laser-based sources [15] and Quantum Cascade Lasers [16,17]. Thus, $\mathrm{THz}$ radiation is now available in both continuous wave $(\mathrm{CW})$ and pulsed form, down to single-cycles and sub-s duration, with peak powers up to tens of MW; several $\mathrm{THz}$ facilities are distributed worldwide for fundamental experiments, user applications, and industrial R\&D.

The rapid advance of $\mathrm{THz}$ technologies in terms of radiation generators, systems, and scientific or industrial applications has put a particular focus on compact sources with challenging performances. Free-electron-laser (FEL)-based sources are probably the best candidates to represent such versatility. There are a number of schemes that have been investigated over the years to generate coherent radiation from free electrons in the $\mathrm{mm}$-wave and $\mathrm{THz}$ regions of the spectrum. The paper by Doria et al. [18] proposes new approaches for exploring the limits in the performance of radio frequency-driven free-electron devices in terms of ultra-short pulse duration, wide bandwidth operation, and energy recovery for near $\mathrm{CW}$ operation. This work demonstrates that a THz FEL is very powerful and flexible. This is possible by tailoring its spectral features with innovative design schemes that can be introduced for the realization of a compact FEL source, as discussed in this contribution.

Considering modern THz sources, TeraFERMI is the THz beamline at the FERMI freeelectron-laser facility in Trieste (Italy). It uses super-radiant Coherent Transition Radiation emission to produce $\mathrm{THz}$ pulses from 10 to $100 \mu \mathrm{J}$ intensity over a spectral range, which can extend up to $12 \mathrm{THz}$. This facility can be used to perform non-linear, fluence-dependent $\mathrm{THz}$ spectroscopy and THz-pump/IR-probe measurements. Adhlakha et al. [19] describe in their contribution the optical set-up in use, and discuss the properties of the generated $\mathrm{THz}$ electric field profile. They showed that the THz emission from TeraFERMI is the combination of two components, and the essential role in the emission properties of this source played by CSR induced wakefields.

$\mathrm{THz}$ radiation is a very appealing band of the electromagnetic spectrum due to its practical applications. In this context, $\mathrm{THz}$ generation and manipulation are an essential part of the technological development. The demand of $\mathrm{THz}$ antennas is still high, as it is difficult to obtain directive, efficient, planar, low-cost, and easy-to-fabricate THz radiating systems. Tofani and Fuscaldo, in their contribution [20], focused on different classes of leaky-wave antennas, based on materials with tunable quasi-optical parameters to produce patterns with particularly high efficiency, taking into account costs and fabrication complexity. Design strategies have been also discussed and some examples have been presented and analyzed.

D'Arco et al., in their contribution [21] measured the complex refraction index of one HMQ-TMS (2-(4-hydroxy-3-methoxystyryl)-1-methylquinolinium 2,4,6-trimethyl benzenesulfonate) single crystal from $\mathrm{THz}$ to $\mathrm{UV}$, observing a remarkable anisotropy, strongly 
attenuated at long wavelengths. The HMQ-TMS is a recently discovered anisotropic organic crystal that can be exploited for the production of broadband high-intensity $\mathrm{THz}$ radiation through the optical rectification technique. It plays a central role in $\mathrm{THz}$ technology due to its broad transparency range, large electro-optic coefficient and coherence length, and excellent crystal properties. The authors extracted both the refraction index $\mathrm{n}$ and the extinction coefficient $\mathrm{k}$ between 50 and $35,000 \mathrm{~cm}^{-1}$, and measured the $\mathrm{THz}$ radiation generated by optical rectification at different wavelengths and along the axes. Data highlighted well the remarkable anisotropic linear and non-linear optical behavior of these crystals, extending the knowledge of their properties from $\mathrm{THz}$ to UV wavelengths and suggesting applications in $\mathrm{THz}$ pump-probe experiments of exotic electronic systems.

The THz electromagnetic characterization of materials is also particularly important in many applications in accelerator physics. As, in storage rings and linear accelerators of new generation, very short bunches might be required, extending the exploration of the beam spectrum far in frequency is required to assess the coating material response up to hundreds of GHz. The paper of Passarelli et al. [22] describes a time domain method based on the waveguide spectroscopy to infer the coating properties at very high frequencies. The approach is extremely useful for the evaluation of the real part of the surface impedance, currently used for modeling the resistive wall component of the beam impedance in accelerators. In fact, the emergence of meta-materials opened new opportunities in many fields including $\mathrm{THz}$. It has been shown that meta-material waveguides can guide a mode, even when the core diameter is more than 10 times smaller than the operating wavelength $[9,23]$.

The incredible scientific and technological advances have recently allowed the production and the coherent detection of sub-ps pulses of $\mathrm{THz}$ radiation, making spectroscopy and imaging in biomedicine at very long wavelengths now possible [24-27]. THz pulsed imaging (TPI) was pioneered in the last decade and D'Arco et al., in their manuscript [28], presented a review of TPI. They discussed the basic principles and performances, and described state-of-the-art applications on biomedical systems. Concerning the $\mathrm{THz}$ radiation properties, they also discussed the major applications of TPI with ex vivo and in vivo studies and cases of histopathological imaging of cancers. Although $\mathrm{THz}$ technology is rapidly expanding as a medical imaging modality, its use requires more attention, and extended trials should be carried out to apply TPI systems in clinical fields and hospitals to transfer this already mature technique for research in a powerful tool for clinical tests [29].

InfraRed synchrotron radiation (IRSR) is a unique broadband light source covering a spectral region from $\mathrm{THz}$ to IR. Its brilliance represents the main advantage for microspectroscopy experiments, perfect to match the demands of extremely small samples that prevent the use of conventional thermal radiation sources [30]. One of the most promising applications of IRSR is cultural heritage, where materials to investigate are unique, delicate, and valuable. A non-destructive approach is mandatory, and extremely small pieces have to be investigated whenever is required, following the logic of preservation and conservation. Ikemoto et al. [31] analyzed several samples at the BL43IR beamline at SPring-8, showing an advantage of more than two orders of magnitude vs. thermal radiation sources and, in particular, in the low-wavenumber region. Iron oxides in bengala red pigment, biomineral distribution caused by lichen growing on a stone, adhesives of fibers of a traditional costume, and organic materials in overglaze layers on ceramics were characterized at high-spatial resolution.

As outlined above, accelerators show other fantastic properties that can be exploited, particularly in the $\mathrm{THz}$ domain. Coherent synchrotron radiation from an electron storage ring is observed in the $\mathrm{THz}$ spectral range when the bunch length is shortened to the sub-mm-range. Schade and co-workers, in their contribution [32], showed that increasing the stored current in the ring causes the bunch to become longitudinally unstable and modulates the $\mathrm{THz}$ emission in the time domain. Their results set the limits for the possible time resolution of pump-probe experiments with coherent $\mathrm{THz}$ synchrotron radiation from a storage ring. Ultra-short electron bunches, such as those delivered by a high-brightness 
photo-injector, are suitable to produce high peak power $\mathrm{THz}$ radiation, both broad and narrow band, with sub-picosecond down to femtosecond pulse shaping. The features of this kind of source are extremely appealing for frequency- and time-domain experiments in a wide variety of fields. The manuscript by Chiadroni et al. [33] will overview the method of generation and characterization of $\mathrm{THz}$ radiation produced by high-brightness electron beams. This radiation can be produced under different regimes, by a proper control of the bunch shaping and charge distribution. Different electron longitudinal distribution can be tailored and manipulated by properly setting the UV photocathode laser and the photoinjector parameters. Data collected at the SPARC_LAB accelerator at the Frascati National Laboratory of the INFN, with both ultra-short single bunch and multi-bunches electron comb beams, provided high energy per pulse and broad and narrow spectral bandwidth $\mathrm{THz}$ radiation, for non-linear and pump-probe experiments in solid-state physics and materials science.

Correlated with this research, the manuscript by Sakai and co-workers [34] is an original study that introduces a new technology to measure electron bunch length at high time resolutions. Using the coherent synchrotron radiation (CSR) emission, they evaluated the RMS bunch lengths in full-bunch mode and in burst mode. CSR was measured by using a narrow-band detector at the S-band linear accelerator facility of the Nihon University. The results can be applied to the precise evaluation of FEL gain vs. charge to develop high-power terahertz light sources.

The demand of new detectors is emerging, correlated with the emergence of new $\mathrm{THz}$ sources [35]. The realization of emitters and receivers is challenging as $\mathrm{THz}$ frequencies are too high for conventional electronics and the photon energies are too small for classical optics. At present, there exists a large variety of $\mathrm{THz}$ radiation sensors and detectors as traditional bolometers, and new devices based on different principles and materials. [36] In his contribution Rezvani et al. presented an innovative photon detector based on the proximity junction array device (PAD) working at long wavelengths. [37] The PAD has a relatively high photo-response, even at frequencies below the expected characteristic frequency, while its superconducting properties such, as the order parameter and the Josephson characteristic frequency, can be easily modulated via external fields to extend the detection band. Authors characterized the vortex dynamics of this proximity array device vs. external parameters, e.g., magnetic and electric fields, showing the occurrence of the transition from a Mott insulating to a metallic state. Advanced devices such as these represent promising detectors for new high-sensitivity long-wavelength systems and for $\mathrm{THz}$ imaging applications.

$\mathrm{THz}$ imaging is one of the many challenging and stimulating $\mathrm{THz}$ applications. Using the unique characteristics of the FEL, Irizawa et al. [38] successfully performed highsensitivity spectral imaging of different materials in the $\mathrm{THz}$ and far-infrared (FIR) domain. Due to the intensity and directionality of the ISIR source at Osaka, they collected high-sensitivity transmission imaging of extremely low-transparency materials and threedimensional objects in the 3-6 THz range. Using the FEL time structure, fast spectral imaging was demonstrated on different samples in various forms. Taking advantage of the available high power, they succeeded in the acquisition of transmission images of "opaque" solid materials and liquids for the first time in the THz/FIR domain. By accurately identifying the intrinsic absorption wavelength of organic and inorganic materials, they also succeeded in the mapping of spatial distribution of individual components. They also showed, again for the first time, that non-linear phenomenon can be observed by changing the FEL intensity, pointing out the huge opportunities of these coherent high-power, high brilliance sources for much interdisciplinary research.

The incredible opportunities offered by intense $\mathrm{THz}$ sources is well represented by the manuscript of Macis et al. [39] which showed the damage induced by an intense coherent $\mathrm{THz}$ beam on copper surfaces. This metallic surface, irradiated by multiple picosecond $\mathrm{THz}$ pulses generated by a FEL, exposed the sample surface to an electric field up to $\sim 4 \mathrm{GV} / \mathrm{m}$. No damage occurs at normal incidence, while images and spectroscopic analysis of the 
surface point out a clear dependence on the incidence angle, the electric field intensity, and polarization of the pulsed $\mathrm{THz}$ radiation. The experimental approach they introduced, with multiple fast irradiations, represents a new powerful technique useful to test the damage induced by an intense electric gradient on metallic surfaces in a reproducible way. This work shows for the first time the occurrence of an angular dependent reproducible damage induced by $\mathrm{THz}$ multiple high-intensity pulses on a metallic surface depending on incidence angle, number of shots, and fluence. The phenomenology behind the observed behavior can be understood in terms of a fast local temperature increase, which may induce ablation and melting on the surface. This original work clearly points out the need to consider electric-related effects, e.g., discharges and/or breakdowns occurring in the air due to the intense applied electric field [40]. The mechanism behind THz-radiation-induced morphological change is also unclear in soft materials. Changes in the intermolecular conformation of bulk materials without damaging the chemical structures have been already observed in bio-macromolecules. Consequently, the use of $\mathrm{THz}$ irradiation may constitute a new method also applicable to biological science, to design and characterize new functional materials [41].

\section{Conclusions}

There is still too much we do not know about working at $\mathrm{THz}$ frequencies and with $\mathrm{THz}$ radiation. We should continue to invest in basic science and technology, and to introduce new computational models for analyzing devices and operation in this energy range. We also need to invest in new methodologies [9] to better understand material properties [42-44] and meta-materials [45] at terahertz frequencies, as well as general terahertz phenomenology. This book deals with emerging applications in $\mathrm{THz}$ solid-state physics and devices such as sources, detectors, advanced materials, and light-matter interactions with theoretical, methodological, well-established, and validated examples: spectroscopic characterization of novel materials, imaging applications in biomedicine, cultural heritage, and earth science [46-49]. It intends to offer results and ideas for a very vast audience, from basic science to engineering, technology experts, newcomers, and students. Indeed, the published contributions describe, in our opinion, an up to date and reliable state-of-art of $\mathrm{THz}$ research and, we hope, their reading will help and promote research on and with $\mathrm{THz}$ radiation.

Author Contributions: A.I., S.L. and A.M. contributed equally to the manuscript. All authors have read and agreed to the published version of the manuscript.

Funding: We acknowledge the financial support of the Bilateral Cooperation Agreement between Italy and Japan of the Italian Ministry of Foreign Affairs and of the International Cooperation (MAECI) in the framework of the project of major relevance N. PGR00728.

Institutional Review Board Statement: Not applicable to the studies discussed in this text.

Informed Consent Statement: Not applicable to these studies discussed in this text.

Conflicts of Interest: The authors declare no conflict of interest.

\section{References}

1. Scalari, G.; Maissen, C.; Cibella, S.; Leoni, R.; Reichl, C.; Wegscheider, W.; Beck, M.; Faist, J. THz ultrastrong light-matter coupling. Il Nuovo Saggiatore 2015, 31, 4-14.

2. Dhillon, S.S.; Vitiello, M.S.; Linfield, E.H.; Davies, A.G.; Hoffmann, M.C.; Booske, J.; Paoloni, C.; Gensch, M.; Weightman, P.; Williams, G.P.; et al. The 2017 terahertz science and technology roadmap. J. Phys. D Appl. Phys. 2017, 50, 043001-043049. [CrossRef]

3. Kawano, Y. Terahertz waves: A tool for condensed matter, the life sciences and astronomy. Contemp. Phys. 2013, 54, 143-165. [CrossRef]

4. Tonouchi, M. Cutting-edge terahertz technology. Nat. Photonics 2007, 1, 97-105. [CrossRef]

5. Mittleman, D.M. Perspective: Terahertz science and technology. J. Appl. Phys. 2017, 122, 230901. [CrossRef]

6. Terahertz Optics. Available online: https://www.nature.com/collections/njscjcdjjr (accessed on 23 June 2021). 
7. Wang, H.B.; Wu, P.H.; Yamashita, T. Terahertz Responses of Intrinsic Josephson Junctions in High $\mathrm{T}_{\mathrm{C}}$ Superconductors. Phys. Rev. Lett. 2001, 87, 107002. [CrossRef] [PubMed]

8. Innocenzi, P.; Malfatti, L.; Marcelli, A.; Piccinini, M.; Sali, D.; Schade, U. Application of Terahertz spectroscopy to time-dependent chemical-physical phenomena. J. Phys. Chem. A 2009, 113, 9418-9423. [CrossRef] [PubMed]

9. Limaj, O.; Giorgianni, F.; Gaspare, A.D.; Giliberti, V.; Marzi, G.D.; Roy, P.; Ortolani, M.; Cunnane, D.; Xi, X.; Lupi, S. Superconductivity-Induced Transparency in Terahertz Metamaterials. ACS Photonics 2014, 1, 570-575. [CrossRef]

10. Zhang, X.C.; Shkurinov, A.; Zhang, Y. Extreme terahertz science. Nat. Photonics 2017, 11, 16-18. [CrossRef]

11. Ma, J.; Shrestha, R.; Adelberg, J.; Yeh, C.-Y.; Hossain, Z.; Knightly, E.; Jornet, J.M.; Mittleman, D.M. Security and eavesdropping in terahertz wireless links. Nature 2018, 563, 89-96. [CrossRef]

12. Gallerano, G.P. Overview of Terahertz Radiation Sources. In Proceedings of the 2004 FEL Conference, Trieste, Italy, 29 August-3 September 2004; pp. 216-221.

13. Chiadroni, E.; Bacci, A.; Bellaveglia, M.; Boscolo, M.; Castellano, M.; Cultrera, L.; Di Pirro, G.; Ferrario, M.; Ficcadenti, L.; Filippetto, D.; et al. The SPARC linear accelerator based terahertz source. Appl. Phys. Lett. 2013, 102, 094101. [CrossRef]

14. Perucchi, A.; Di Mitri, S.; Penco, G.; Allaria, E.; Lupi, S. The TeraFERMI terahertz source at the seeded FERMI free-electron-laser facility. Rev. Sci. Instrum. 2013, 84, 022702. [CrossRef]

15. Fülöp, J.A.; Tzortzakis, S.; Kampfrath, T. Laser-Driven Strong-Field Terahertz Sources. Adv. Opt. Mater. 2020, 8, 1900681. [CrossRef]

16. Williams, B.S. Terahertz quantum-cascade lasers. Nat. Photonics 2007, 1, 517-525. [CrossRef]

17. Vitiello, M.S.; Tredicucci, A. Physics and technology of Terahertz quantum cascade lasers. Adv. Phys. X 2021, 6, 1893809. [CrossRef]

18. Doria, A.; Gallerano, G.P.; Giovenale, E. Novel Schemes for Compact FELs in the THz Region. Condens. Matter 2019, 4, 90. [CrossRef]

19. Adhlakha, N.; Di Pietro, P.; Piccirilli, F.; Cinquegrana, P.; Di Mitri, S.; Sigalotti, P.; Spampinati, S.; Veronese, M.; Lupi, S.; Perucchi, A. The TeraFERMI Electro-Optic Sampling Set-Up for Fluence-Dependent Spectroscopic Measurements. Condens. Matter 2020, 5, 8. [CrossRef]

20. Tofani, S.; Fuscaldo, W. Fabry-Perot Cavity Leaky Wave Antennas with Tunable Features for Terahertz Applications. Condens. Matter 2020, 5, 11. [CrossRef]

21. D'Arco, A.; Tomarchio, L.; Dolci, V.; Di Pietro, P.; Perucchi, A.; Mou, S.; Petrarca, M.; Lupi, S. Broadband Anisotropic Optical Properties of the Terahertz Generator HMQ-TMS Organic Crystal. Condens. Matter 2020, 5, 47. [CrossRef]

22. Passarelli, A.; Koral, C.; Masullo, M.R.; Vollenberg, W.; Lain Amador, L.; Andreone, A. Sub-THz Waveguide Spectroscopy of Coating Materials for Particle Accelerators. Condens. Matter 2020, 5, 9. [CrossRef]

23. Atakaramians, S.; Afshar, S.; Monro, T.M.; Abbott, D. Terahertz dielectric waveguides. Adv. Opt. Photonics 2013, 5, 169-215. [CrossRef]

24. Choi, H.; Son, J.-H. Terahertz Imaging and Tomography Techniques, Chapt. 4. In Terahertz Biomedical Science and Technology; Son, J.-H., Ed.; CRC Press: Boca Raton, FL, USA, 2014; pp. 47-66.

25. Mittleman, D.M. Twenty years of terahertz imaging. Opt. Express 2018, 26, 9417. [CrossRef]

26. Baxter, J.B.; Guglietta, G.W. Terahertz Spectroscopy. Anal. Chem. 2011, 83, 4342-4368. [CrossRef] [PubMed]

27. McIntosh, A.I.; Yang, B.; Goldup, S.M.; Watkinson, M.; Donnan, R.S. Terahertz spectroscopy: A powerful new tool for the chemical sciences? Chem. Soc. Rev. 2012, 21, 8210. [CrossRef] [PubMed]

28. D'Arco, A.; Di Fabrizio, M.; Dolci, V.; Petrarca, M.; Lupi, S. THz Pulsed Imaging in Biomedical Applications. Condens. Matter 2020, 5, 25. [CrossRef]

29. Yu, C.; Fan, S.; Sun, Y.; Pickwell-MacPherson, E. The potential of terahertz imaging for cancer diagnosis: A review of investigations to date. Quant. Imaging Med. Surg. 2012, 2, 33-45. [CrossRef]

30. Marcelli, A.; Cinque, G. Infrared synchrotron radiation beamlines: High brilliance tools for IR spectromicroscopy. A practical guide to the characteristics of the broadband and brilliant non-thermal sources. In Biomedical Applications of Synchrotron Infrared Microspectroscopy; Moss, D., Ed.; Royal Society of Chemistry: London, UK, 2011; Chapter 3; pp. 67-104.

31. Ikemoto, Y.; Tanaka, M.; Higuchi, T.; Semba, T.; Moriwaki, T.; Kawasaki, E.; Okuyama, M. Infrared Synchrotron Radiation and Its Application to the Analysis of Cultural Heritage. Condens. Matter 2020, 5, 28. [CrossRef]

32. Schade, U.; Kuske, P.; Lee, J.; Marchetti, B.; Ortolani, M. Cross-Correlation of THz Pulses from the Electron Storage Ring BESSY II. Condens. Matter 2020, 5, 24. [CrossRef]

33. Chiadroni, E.; Cianchi, A.; Ferrario, M.; Mostacci, A.; Pompili, R.; Shpakov, V. A Versatile THz Source from High-Brightness Electron Beams: Generation and Characterization. Condens. Matter 2020, 5, 40. [CrossRef]

34. Sakai, T.; Hayakawa, K.; Tanaka, T.; Hayakawa, Y.; Nogami, K.; Sei, N. Evaluation of Bunch Length by Measuring Coherent Synchrotron Radiation with a Narrow-Band Detector at LEBRA. Condens. Matter 2020, 5, 34. [CrossRef]

35. Rogalski, A.; Sizov, F. Terahertz detectors and focal plane arrays. Opto-Electron. Rev. 2011, 19, 346-404. [CrossRef]

36. Sizov, F. THz radiation sensors. Opto-Electron. Rev. 2010, 18, 10-36. [CrossRef]

37. Rezvani, S.J.; Gioacchino, D.D.; Gatti, C.; Ligi, C.; Guidi, M.C.; Cibella, S.; Fretto, M.; Poccia, N.; Lupi, S.; Marcelli, A. Proximity Array Device: A Novel Photon Detector Working in Long Wavelengths. Condens. Matter 2020, 5, 33. [CrossRef] 
38. Irizawa, A.; Fujimoto, M.; Kawase, K.; Kato, R.; Fujiwara, H.; Higashiya, A.; Macis, S.; Tomarchio, L.; Lupi, S.; Marcelli, A.; et al. Spatially Resolved Spectral Imaging by a THz-FEL. Condens. Matter 2020, 5, 38. [CrossRef]

39. Macis, S.; Tomarchio, L.; Tofani, S.; Rezvani, S.J.; Faillace, L.; Lupi, S.; Irizawa, A.; Marcelli, A. Angular Dependence of Copper Surface Damage Induced by an Intense Coherent THz Radiation Beam. Condens. Matter 2020, 5, 16. [CrossRef]

40. Agranat, M.B.; Chefonov, O.V.; Ovchinnikov, A.V.; Ashitkov, S.I.; Fortov, V.E.; Kondratenko, P.S. Damage in a Thin Metal Film by High-Power Terahertz Radiation. Phys. Rev. Lett. 2018, 120, 085704. [CrossRef] [PubMed]

41. Hoshina, H.; Suzuki, H.; Otani, C.; Nagai, M.; Kawase, K.; Irizawa, A.; Isoyama, G. Polymer Morphological Change Induced by Terahertz Irradiation. Sci. Rep. 2016, 6, 27180. [CrossRef]

42. Williams, C.R.; Andrews, S.R.; Maier, S.A.; Fernandez-Dominguez, A.I.; Martin-Moreno, L.; Garcia-Vidal, F.J. Highly confined guiding of terahertz surface plasmon polaritons on structured metal surfaces. Nat. Photonics 2008, 2, 175-179. [CrossRef]

43. D'Apuzzo, F.; Piacenti, A.R.; Giorgianni, F.; Autore, M.; Cestelli Guidi, M.; Marcelli, A.; Schade, U.; Ito, Y.; Chen, M.; Lupi, S. Terahertz and Mid-Infrared Plasmons in 3-Dimensional Nanoporous Graphene. Nat. Commun. 2017, 8, 14885. [CrossRef] [PubMed]

44. Sacchetti, A.; Cestelli Guidi, M.; Arcangeletti, E.; Nucara, A.; Calvani, P.; Piccinini, M.; Marcelli, A.; Postorino, P. Far-infrared absorption of $\mathrm{La}_{1-x} \mathrm{Ca}_{x} \mathrm{MnO}_{3-y}$ at high pressure. Phys. Rev. Lett. 2006, 96, 035503. [CrossRef] [PubMed]

45. Pendry, J.B.; Holden, A.; Robbins, D.; Stewart, W. Magnetism from conductors and enhanced nonlinear phenomena. IEEE Trans. Microw. Theory Tech. 1999, 47, 2075-2084. [CrossRef]

46. Cinque, G.; Marcelli, A. Synchrotron Radiation InfraRed microspectroscopy and imaging in the characterization of archaeological materials and cultural heritage artefacts. In EMU Notes in Mineralogy; European Mineralogical Union and the Mineralogical Society of Great Britain \& Ireland: London, UK, 2019; Volume 20, Chapter 12; pp. 411-444.

47. Della Ventura, G.; Bellatreccia, F.; Marcelli, A.; Cestelli Guidi, M.; Piccinini, M.; Cavallo, A.; Piochi, M. Application of micro-FTIR imaging in Earth Sciences. Anal. Bioanal. Chem. 2010, 397, 2039-2049. [CrossRef]

48. Della Ventura, G.; Marcelli, A.; Bellatreccia, F. SR-FTIR microscopy and FTIR imaging in the Earth Sciences. Rev. Mineral. Geochem. 2014, 78, 447-479. [CrossRef]

49. Valušis, G.; Lisauskas, A.; Yuan, H.; Knap, W.; Roskos, H.G. Roadmap of Terahertz Imaging 2021. Sensors 2021, $21,4092$. [CrossRef] 